\title{
A complete radio study of SNR G15.4+0.1 from new GMRT observations
}

\author{
L. Supan ${ }^{1}$, G. Castelletti ${ }^{1}$, B. C. Joshi ${ }^{2}$, M. P. Surnis ${ }^{2}$, and D. Supanitsky ${ }^{1}$ \\ ${ }^{1}$ Instituto de Astronomía y Física del Espacio (IAFE, CONICET-UBA), CC 67, Suc. 28, 1428 Buenos Aires, Argentina \\ e-mail: 1supan@iafe.uba.ar \\ 2 National Centre for Radio Astrophysics (NCRA-TIFR), Post Bag No 3, Ganeshkhind, 411007 Pune , India
}

Received 5 November 2014 / Accepted 16 January 2015

\begin{abstract}
Aims. The supernova remnant (SNR) G15.4+0.1 is considered to be the possible counterpart of the $\gamma$-ray source HESS J1818-154. With the goal of getting a complete view of this remnant and understanding the nature of the $\gamma$-ray flux, we conducted a detailed radio study that includes the search for pulsations and a model of the broadband emission for the SNR G15.4+0.1/HESS J1818-154 system.

Methods. Low-frequency imaging at $624 \mathrm{MHz}$ and pulsar observations at 624 and $1404 \mathrm{MHz}$ towards G15.4+0.1 were carried out with the Giant Metrewave Radio Telescope (GMRT). We correlated the new radio data with observations of the source at X-ray and infrared wavelengths from XMM-Newton and Herschel observatories, respectively. To characterize the neutral hydrogen (HI) medium towards G15.4+0.1, we used data from the Southern Galactic Plane Survey. We modelled the spectral energy distribution (SED) using both hadronic and leptonic scenarios.

Results. From the combination of the new GMRT observations with existing data, we derived a continuum spectral index $\alpha=$ $-0.62 \pm 0.03$ for the whole remnant. The local synchrotron spectra of G15.4+0.1, calculated from the combination of the GMRT data with $330 \mathrm{MHz}$ observations from the Very Large Array, tends to be flatter in the central part of the remnant, accompanying the region where the blast wave is impinging molecular gas. No spectral index trace was found indicating the radio counterpart to the pulsar wind nebula proposed from X-ray observations. In addition, the search for radio pulsations yielded negative results. Emission at far-infrared wavelengths is observed in the region where the SNR shock is interacting with dense molecular clumps. We also identified $\mathrm{HI}$ features forming a shell that wraps most of the outer border of G15.4+0.1. Characteristic parameters were estimated for the shocked HI gas. We found that either a purely hadronic or leptonic model is compatible with the broadband emission known so far.
\end{abstract}

Key words. ISM: individual objects: SNR G15.4+0.1 - ISM: supernova remnants - radio continuum: ISM - gamma rays: ISM

\section{Introduction}

The radio source G15.4+0.1 was first identified as a supernova remnant (SNR) during the $90 \mathrm{~cm}$ multiconfiguration Very Large Array (VLA) survey of the Galactic plane (Brogan et al. 2006). This remnant is found to positionally coincide with extended emission ( $~ 8.5$ arcmin in size) detected in the $\mathrm{TeV} \gamma$-ray band with the High Energy Stereoscopic System (HESS) (Hofverberg et al. 2011). The TeV source, catalogued as HESS J1818-154, is one of the faintest HESS sources detected to date. On the basis of the morphological correspondence between the brightest hotspot of HESS J1818-154 and the inner part of G15.4+0.1, it has been proposed that a pulsar wind nebula (PWN) powered by a still undetected rotating neutron star could be responsible for the $\mathrm{TeV} \gamma$ rays (Hofverberg et al. 2011).

Recently, Castelletti et al. (2013, hereafter C13) found morphological and kinematical evidence for shocked CO gas in a dense molecular cloud located in the foreground of the SNR G15.4+0.1. Because of the location of the cloud (partially overlapping the HESS source) and the signatures of interaction between the molecular gas and the remnant, the authors argued that the gamma radiation might be arising from hadronic interactions produced by the passage of the supernova shock front through the molecular cloud. In that paper, based on $21 \mathrm{~cm} \mathrm{HI}$ absorption measurements along the line of sight to G15.4+0.1, those authors also redetermined the distance to this remnant placing it at $4.8 \pm 1.0 \mathrm{kpc}$, instead of the $10 \pm 3 \mathrm{kpc}$ estimated by Hofverberg et al. (2011) using the uncertain $\Sigma-\Delta$ relation for supernova remnants.

Later on, Abramowski et al. (2014) used the XMM-Newton telescope to investigate the X-ray counterpart to the $\gamma$-ray source HESS J1818-154. The new X-ray image of the remnant shows extended emission in the interior of the radio SNR shell, in spatial coincidence with the discovered HESS source and the southern part of the molecular cloud. Although the X-ray emission is fitted satisfactorily by both the thermal and non-thermal models, these authors interpreted the X-rays as emission from a PWN. In addition, the X-ray observations lead to the detection of five point-like sources in the region of the $\gamma$-ray emission. From their X-ray properties, only two were thought to be galactic sources that could be candidates for the central compact object of the SNR. The analysis of periodicity in the signal from these sources, however, has not shown evidence of X-ray pulsations.

In this paper, based on new data obtained with the Giant Metrewave Radio Telescope (GMRT), we provide the first comprehensive analysis of the morphological and spectral radio properties of SNR G15.4+0.1, which includes the results of the search for radio pulsations in the region of the source 
HESS J1818-154. In addition, we report on the study of the spatial correspondences between the radio, infrared, and $\mathrm{X}$-ray emission bands, and the analysis of the neutral hydrogen distribution in the environment of the remnant. On the basis of the flux measurements from radio to $\gamma$ ray, we discuss scenarios in which the $\mathrm{TeV}$ emission from this source originates from either hadrons interacting with dense interstellar material or leptonic emission.

\section{Observations and imaging}

\subsection{Radio continuum and neutral hydrogen observations}

The SNR G15.4+0.1 was observed using the GMRT at $624 \mathrm{MHz}$ on June 17 and 18, 2012 (Project code 22-015). The project also includes GMRT observations of the remnant at $1420 \mathrm{MHz}$. The image at this frequency was presented for the first time in C13, and so it is not shown again here. The data were collected in a total bandwidth of $33.33 \mathrm{MHz}$ split into 512 spectral channels. The flux density calibrator used was 3C 286, for which a flux density of 14.92 Jy was set using the Perley-Butler 2010 VLA values ${ }^{1}$. Regular observations of the source 1822-096 were used for phase and bandpass calibration. Reduction and imaging were carried out with the NRAO Astronomical Image Processing System (AIPS) package. The data from each day were fully reduced separately and then combined in a single data set. The reduction of the data consisted of the removal of radio frequency interference (RFI) by hand rather than with the automatic flagging routines, and bandpass and gain calibrations. After initial calibration, the data were averaged in terms of frequency by collapsing the bandwidth to a number of 100 spectral channels. For the concatenated $624 \mathrm{MHz}$ data set, we employed wide-field imaging based on a pseudo-three-dimensional multifacet algorithm. In addition, we used a multi-scale CLEAN algorithm in AIPS, with four different scale sizes. Several selfcalibration and imaging iterations were made to obtain the final image. The resulting GMRT image at $624 \mathrm{MHz}$ has an angular

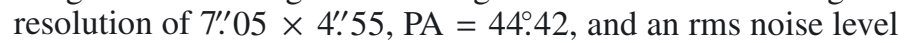
of $0.15 \mathrm{mJy}$ beam $^{-1}$. We note the significantly high sensitivity (a factor of 10 better than the published image at $330 \mathrm{MHz}$ ) of this image at $624 \mathrm{MHz}$, being reported for the first time.

On the other hand, the interstellar medium (ISM) in the direction of G15.4+0.1 was investigated using data from the Southern Galactic Plane Survey in the $21 \mathrm{~cm}$ emission line produced by HI (SGPS, McClure-Griffiths et al. 2005). The SGPS images, combining data from the Australia Telescope Compact Array (ATCA) and the Parkes $64 \mathrm{~m}$ single-dish telescope, are valuable in tracing large scale $\mathrm{HI}$ structures with an angular resolution of $3.3 \times 2 ! 1, \mathrm{PA}=135^{\circ}$, and a velocity channel spacing of $0.82 \mathrm{~km} \mathrm{~s}^{-1}$.

\subsection{Radio time-series observations}

Time-series observations towards the reported centroid of the HESS source, $l=15^{\circ} .41, b=0.17$ (Hofverberg et al. 2011), were carried out with the GMRT at $1404 \mathrm{MHz}$ on May 11 and 12, 2012, and at $624 \mathrm{MHz}$ on June 17 and 18, 2012 (Project code 22-015), simultaneously with the imaging observations. At each frequency, the GMRT was simultaneously used in two different modes to obtain two different time-series. In one mode,

\footnotetext{
1 For more detailed information regarding the flux density calibrators at VLA see the explanation of AIPS task SETJY or the website at http://www.nrao.edu/doc/vla/html/calib.shtml
}

the output of 26 antennas were combined in an incoherent array (IA) with an effective beam of $43^{\prime}$ and $24^{\prime}$ at 624 and $1404 \mathrm{MHz}$, respectively. The five X-ray point sources, identified in the XMM-Newton observations (Abramowski et al. 2014), all lie between $2^{\prime}-5^{\prime}$ from the centre of this beam and were covered in the search for pulsation. A higher sensitivity phased array (PA) of 15 antennas, with an effective beam of $100^{\prime \prime}$ and $40^{\prime \prime}$ at 624 and $1404 \mathrm{MHz}$, respectively, and pointed at the centroid of the HESS position, was used in the other mode. Both time-series were sampled every $61 \mu$ s with 512 channels across a $33.33 \mathrm{MHz}$ bandpass at each frequency of observation. To accomplish the periodic requirement of phase calibration for both imaging and phased array observations, the time-series were acquired in 13 non-contiguous integrations of about 20 minute duration each at 624 and $1404 \mathrm{MHz}$, respectively. In order to assess the sensitivity of the search for radio pulsations, as well as the dispersion measures (DM) and the flux density estimates, we also carried out time-series observations in direction of three known pulsars: B1642-03, B1937+21, and J1901-0906.

\subsection{X-rays observations}

With the aim of performing a multiwavelength analysis of the emission from G15.4+0.1, we reprocessed data extracted from the on-line archive of the XMM-Newton observations ${ }^{2}$. The observations in the direction of the remnant were made on October 10, 2012, during revolution 2351 (Obs-ID: 0691390101).

The data were reduced using the XMM-Newton Science Analysis System (SAS) software package Version 13.5.0. We applied standard processing to this data set to obtain clean event files. In order to mitigate the impact of high background flare activity, an appropriate screening was applied, extracting a light curve for photons above $10 \mathrm{keV}$ for the entire field of view. We selected events with FLAG $=0$ and PATTERN $\leqslant 12$ for both EPIC MOS1 and MOS2 cameras (Turner et al. 2001). The resulting total effective exposure time of observation with the MOS1 and MOS2 cameras was $30 \mathrm{ks}$.

In order to reveal the diffuse emission, point-like sources within the field of view were extracted by using the dmfilth tool of the CIAO (v. 4.6) reduction package ${ }^{3}$ with POISSON statistics to fill the excluded regions. Finally, a single combined image in the energy range $1-8 \mathrm{keV}$ from both cameras was obtained, which was then smoothed with a Gaussian kernel to a resolution of $\sim 1$. 4 .

\section{Results}

\subsection{Total intensity image of $\mathrm{G} 15.4+0.1$ at $624 \mathrm{MHz}$}

Figure 1 shows the new image of SNR G15.4+0.1 obtained at $624 \mathrm{MHz}$ using the GMRT. It constitutes the first ever highresolution and sensitivity image of this source at low radio frequencies. The shortest baseline available in the GMRT observations at $624 \mathrm{MHz}$ is $\sim 197 \lambda$, which implies that spatial scales of the G15.4+0.1 emission up to $\sim 17.4$ arcmin are well sampled in the resulting image. The radio emission from this remnant has the appearance of an irregular shell somewhat elongated in the

2 XMM-Newton Science Archive (XSA) http://xmm.esac.esa. int/xsa/index. shtml

3 More information available at http://cxc . harvard.edu/ciao/ 


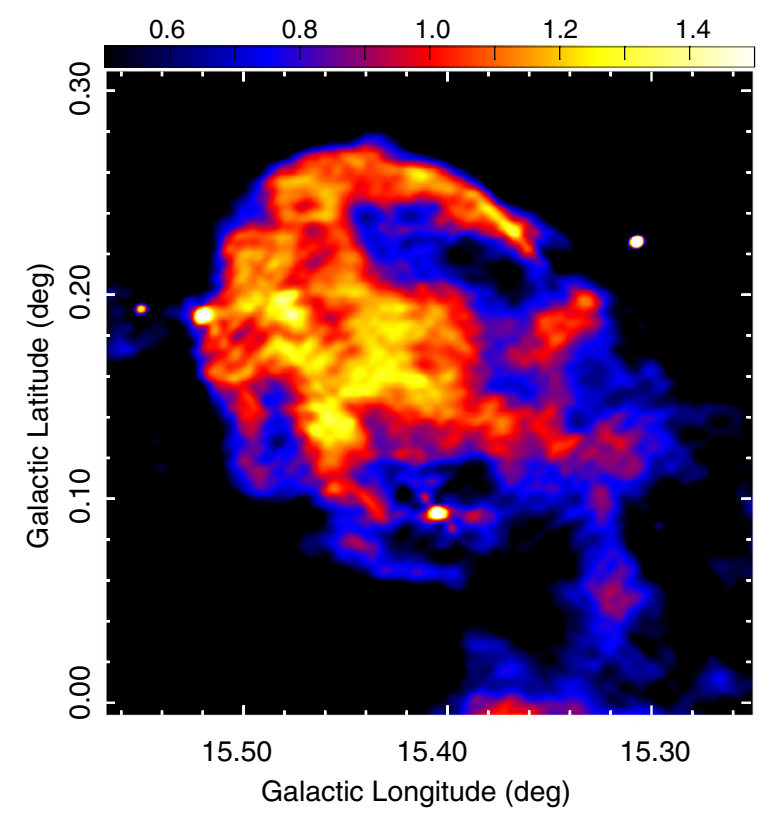

Fig. 1. Total intensity image of SNR G15.5+0.1 at $624 \mathrm{MHz}$ obtained with the GMRT. It does not include primary beam correction. The image was smoothed to an angular resolution of $10^{\prime \prime}$, resulting in an rms noise level of $0.48 \mathrm{mJy}$ beam $^{-1}$. The unit of the values in the colour bar is mJy beam ${ }^{-1}$.

Galactic north-east to south-west ${ }^{4}$ direction (i.e. from upper left to lower right of Fig. 1) with an average diameter of 14.5 arcmin (i.e. $\sim 20 \mathrm{pc}$ at the distance of $4.8 \mathrm{kpc}$ derived in C13). The same overall total intensity morphology is also observed in the images of this remnant at 330 and $1420 \mathrm{MHz}$ reported by Brogan et al. (2006) and in C13 based on VLA and GMRT observations, respectively. At $624 \mathrm{MHz}$, the emission is brighter and patchy along the Galactic northwestern rim and from the northeastern to the central part of the radio shell, overlapping the region where shocked CO gas has been identified (C13); see also Fig. 4 in current work. The bright spot at $l=15.52, b=0.19$ corresponds to the $624 \mathrm{MHz}$ radio counterpart of the bipolar bubble HII region G015.520+0.188 (Anderson et al. 2011), for which Supan et al. (2014) have determined a distance of $3.0 \pm 0.3 \mathrm{kpc}$ based on the new GMRT observations and HI emission line data.

The radio emission becomes fainter and uniform towards the southern part of G15.4+0.1. Unlike the north edge, no sharp boundary is apparent in the south limb of the remnant. A gap in the radio emission is also evident along the western edge of the remnant.

To accurately determine the flux density integrated over the whole $624 \mathrm{MHz}$ emission from G15.4+0.1, we subtracted the contribution of the aforementioned HII region and the bright knot located at $l \sim 15.48, b \sim 0.20$, which is likely to be an extragalactic source, previously identified as NVSS J181806-152321 (NED database) ${ }^{5}$. The integrated flux density for G15.4+0.1 based on the new GMRT observations at $624 \mathrm{MHz}$ is $8.0 \pm 1.1 \mathrm{Jy}$ using the Perley \& Butler (2013) flux density scale. The error in our estimate reflects the uncertainty

\footnotetext{
4 Although we do not utilize the equatorial coordinate system, we refer hereafter to north and south to set out locations around the Galactic plane.

5 NED (the NASA/IPAC Extragalactic Database) is operated by the Jet Propulsion Laboratory, California Institute of Technology, under contract with the National Aeronautics and Space Administration.
}

both in the determination of background emission and in the selection of the area over which we integrate the remnant flux.

\subsection{Pulsar search}

The time-series data for both the incoherent and coherent array for each frequency were analyzed using SIGPROC ${ }^{6}$ pulsar data analysis software using a 16 core 32-node High Performance Computing cluster (HPC) at the National Centre for Radio Astrophysics (NCRA, India). First, the channels affected by RFI were flagged from the data. The usable bandwidth of the observations was somewhat less than $33.33 \mathrm{MHz}$ owing to the presence of narrowband RFI channels, which were removed, and was different for incoherent and phased array observations. These were then dedispersed to 1792 trial dispersion measures ${ }^{7}$ (DMs) covering a range of $0-815 \mathrm{pc} \mathrm{cm}^{-3}$ at $624 \mathrm{MHz}$. The DMs were spaced more coarsely at $1404 \mathrm{MHz}$, owing to a small amount of dispersion smear across each channel. Data were dedispersed to 360 trial DMs at this frequency covering $0-1200 \mathrm{pc} \mathrm{cm}^{-3}$. A harmonic search was performed on the dedispersed timeseries for each DM. Because of the non-contiguous nature of the time-series, an incoherent addition of spectra from individual time-series segments was carried out before the harmonic search. Known RFI periodicities, such as power-line frequencies at 50 and $100 \mathrm{~Hz}$, were excised from the resultant spectra before the harmonic search. The resultant candidate periodicities were carefully examined using a visual representation of the search results as shown in Fig. 2. The search typically produced 60 to 70 candidates at each frequency for the phased array observations, while the number of candidates for the incoherent array were larger. No pulsations above a threshold signal-to-noise ratio $(\mathrm{S} / \mathrm{N})$ of 8 were detected in either phased array or incoherent array observations at both frequencies.

The sensitivity of the pulsation search was assessed using observations of the three known pulsars, PSRs B1642-03, B1937+21, and J1901-0906, as mentioned in Sect. 2.2. A procedure similar to that outlined in Cañellas et al. (2012) was used for this purpose. The sensitivity of pulsation search depends on the duty cycle (ratio of pulse width to pulsar period) of the pulsar. As SNR G15.4+0.1 lies in the direction of the Galactic centre, any associated pulsar is likely to be scatter-broadened, particularly at $624 \mathrm{MHz}$, which affects the duty cycle of the pulsar. For example, using NE2001 models (Cordes \& Lazio 2002) with a distance of $4.8 \mathrm{kpc}$ to the SNR (C13), we get an estimate of temporal broadening of about $13 \mathrm{~ms}$ at $624 \mathrm{MHz}$, whereas this value is about $300 \mu$ s at $1404 \mathrm{MHz}$. Hence, we have plotted our sensitivity curves as a function of duty cycle in Fig. 3 to provide a more meaningful comparison in the presence of temporal broadening. The individual curves correspond to the incoherent array and the phased array observations at 624 and $1404 \mathrm{MHz}$, respectively. These curves provide 8 standard deviation upper limits, for pulsed emission with $10 \%$ duty cycle, of 250 and $300 \mu \mathrm{Jy}$ in the phased array observations at 624 and $1404 \mathrm{MHz}$, respectively, from any putative pulsar associated with the SNR G15.4+0.1/HESS J1818-154 system. Upper limits of 700 and $500 \mu \mathrm{Jy}$ in the incoherent array observations at 624 and $1404 \mathrm{MHz}$, respectively, are also implied for the five identified X-ray point sources within the extent of the SNR. We note that the incoherent array beam covers the entire remnant.

\footnotetext{
6 http://sigproc.sourceforge.net

7 The integrated electron column density along the line of sight in units of $\mathrm{pc} \mathrm{cm}^{-3}$.
} 

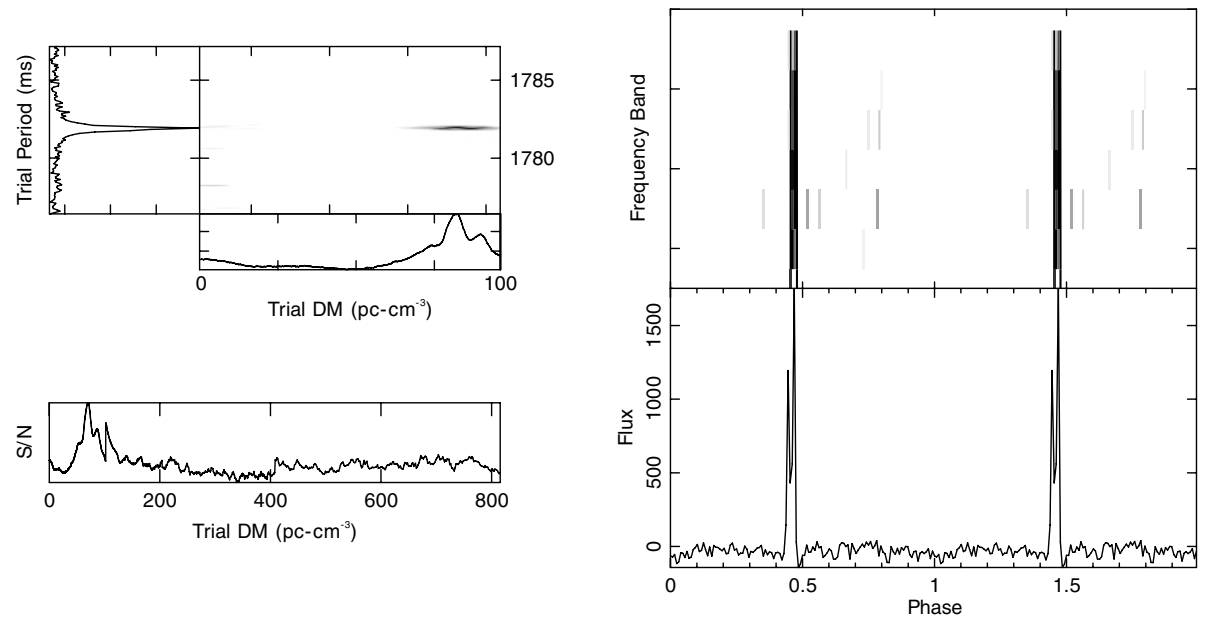

Fig. 2. Visual representation of a pulsar search candidate. The plot shows the detection of the known pulsar PSR J1901-0906, following the procedure described in the text. The bottom left panel shows the peak in the $\mathrm{S} / \mathrm{N}$ curve over a large range of dispersion measures (DM) values, whereas the top left panel indicates the peak in the $\mathrm{S} / \mathrm{N}$ with a refined period and DM. The top right plot shows a greyscale representation of intensity as a function of pulse phase in abscissa and the bandpass in ordinate at the refined period and DM, while the bottom right plot shows the averaged profile of the pulsar. Two rotations are shown for clarity in this plot. This representation helps distinguish mistaken RFI candidates from genuine candidate pulsars.

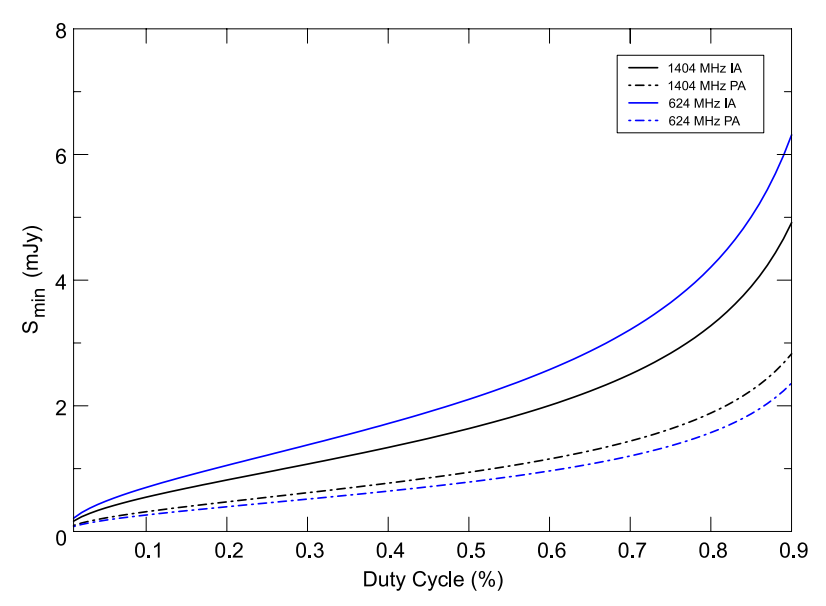

Fig. 3. Sensitivity curves for the pulsational search. The plot depicts the 8 standard deviation upper limits obtained from our observations for incoherent array at $1404 \mathrm{MHz}$ (solid black), phased array at $1404 \mathrm{MHz}$ (dashed-dot black), incoherent array at $624 \mathrm{MHz}$ (solid blue), and phased array at $624 \mathrm{MHz}$ (dashed-dot blue) as a function of duty cycle for a possible pulsar.

\subsection{A multiwavelength view of SNR G15.4+0.1}

We have explored the spatial co-existence of synchrotron radio features and sites of emission at infrared and X-ray wavelengths. In order to compare radio and far-infrared (FIR) morphologies, we made use of archival data at 70 and $250 \mu \mathrm{m}$ acquired using the SPIRE instrument on Herschel Space Observatory ${ }^{8}$ (Griffin et al. 2010). Far-infrared images are a very useful tool with which to delineate shock heated dust, particularly in regions where the SNR shock front is hitting dense ISM. The spatial correspondence between the new radio image at $624 \mathrm{MHz}$ and the FIR images is shown in Fig. 4a; yellow contours are included to help locate the molecular cloud discovered by $\mathrm{C} 13$. To perform the radio/IR comparison, the $70 \mu \mathrm{m}$ data were convolved to the same angular resolution as the $250 \mu \mathrm{m}$ map (18"). From Fig. 4a, the correlation between the $\mathrm{CO}$ molecular cloud and the spatial distribution of the emission at IR wavelengths is clearly evident. Indeed, the elongated structures that appear in the FIR images is completely bounded within the dense $\mathrm{CO}$ structure detected at velocities between 46 and $50.3 \mathrm{~km} \mathrm{~s}^{-1}$ (C13). Moreover,

\footnotetext{
8 Herschel is an ESA space observatory with science instruments provided by European-led Principal Investigator consortia and with important participation from NASA.
}

significant enhancements in the dust emission at the two wavebands are located inside the two molecular clumps (referred to in $\mathrm{C} 13$ as clumps A and B). Such coupling between the dust emission in the FIR and the $\mathrm{CO}$ line emission provides additional support to the presence of dense molecular gas associated with the SNR. We thus interpret the infrared emission as marking the SNR-molecular cloud interface in G15.4+0.1. Evidence of a similar situation has also been observed in the northern shock front of Cas A, in the region where the remnant encounters a density enhancement in the ISM (Hines et al. 2004).

To obtain a more complete description of the infrared distribution in G15.4+0.1, we also used data at 3.6, 4.5, 5.8, and $8 \mu \mathrm{m}$ taken with the IRAC camera aboard the Spitzer Space Telescope along with MIPSGAL images at $24 \mu \mathrm{m}$. Aside from the striking correspondence observed at FIR wavebands with the CO cloud, no discernible emission was detected in the near- or mid-infrared wavebands, which correlates with either the radio surface brightness or the proposed PWN in X-rays (the comparisons are not shown here). This result contrasts with that observed in several PWNe in the Galaxy, for which there is a spatial agreement between synchrotron radio, X-rays, and near-infrared emission distribution (see e.g. the multiwavelength view of the PWNe associated with SNRs 3C 58, Slane et al. 2008; Crab, Sandberg \& Sollerman 2009; G21.5-0.9, Zajczyk et al. 2012; and G292.0+1.8, Zharikov et al. 2013).

The comparison of the emission in radio and X-ray bands was first presented by Abramowski et al. (2014) using the image of the remnant taken from the $330 \mathrm{MHz}$ VLA survey of the Galactic plane (GPS, Brogan et al. 2005a) and XMM-Newton observations. Here, we redo this analysis in the light of the new GMRT data by combining the synchrotron emission at $624 \mathrm{MHz}$ from the source, which shows a highly structured interior compared to that observed at the lower radio frequency, with $X M M-N e w t o n$ reprocessed data obtained from X-ray energies between 1.0 and $8.0 \mathrm{keV}$. The radio/X-ray morphological correlation is shown in Fig. 4b. Contours for the molecular gas interacting with the SNR (C13) together with the region where $\gamma$-ray emission was observed are also presented in the radio/X-ray comparison. Fig. 4b reveals little correspondence between both emitting plasmas. The X-rays associated with G15.4+0.1 do not show evidence of a limb-brightened X-ray shell, but most of the $\mathrm{X}$-ray emission appears broadly contained within the radio rims of G15.4+0.1. Moreover, the comparison shows X-ray emission extending slightly beyond the projected radio boundaries of the remnant. In addition, weak and diffuse X-ray emission is present 


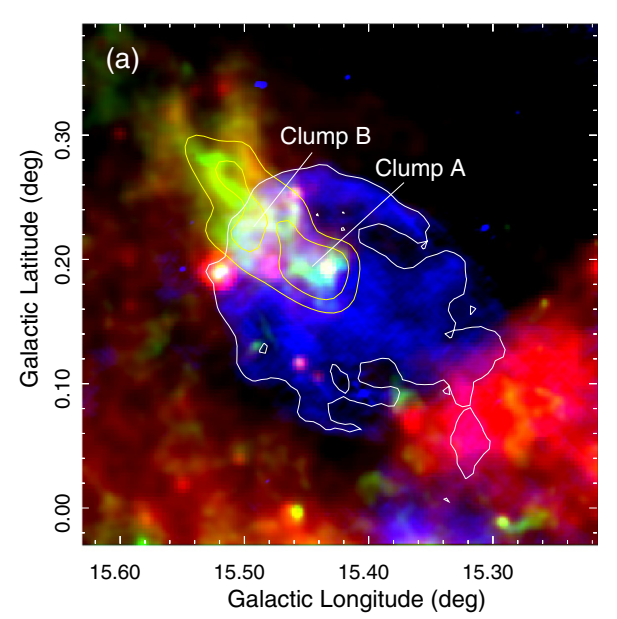

Table 1. Integrated flux densities for G15.4+0.1.

\begin{tabular}{lcc}
\hline \hline $\begin{array}{l}\text { Frequency } \\
(\mathrm{MHz})\end{array}$ & $\begin{array}{c}\text { Scaled } \\
\text { flux density (Jy) }\end{array}$ & References \\
\hline 330 & $11.3 \pm 0.3$ & Brogan et al. (2006) \\
624 & $8.0 \pm 1.1$ & This work \\
1400 & $4.7 \pm 0.8$ & Brogan et al. (2006) \\
1420 & $4.7 \pm 0.2$ & C13 \\
2700 & $2.9 \pm 0.3$ & Brogan et al. (2006) \\
\hline
\end{tabular}

filling the spatial gap observed in radio all along the western limb of the remnant.

Figure $4 \mathrm{~b}$ also demonstrates a significant lack of correspondence between the $\mathrm{X}$-rays and the molecular material, delineated by yellow contours. This result may not be surprising if obscuration due to the molecular cloud detected between the observer and the remnant occurs. On the contrary, the morphology in $\mathrm{X}$-rays overlaps part of the $\gamma$-ray emission area delineated by the white circle in Fig. 4b. Such a X-ray/ $\gamma$ ray correlation led Abramowski et al. (2014) to the conclusion that the X-ray emission is the counterpart to the $\mathrm{TeV}$ source originating in a pulsar wind nebula of a yet undiscovered pulsar. This interpretation, however, may not have a robust observational support since the authors remark that both thermal and non-thermal models are equally satisfactory to fit the spectrum of the diffuse X-rays in G15.4+0.1, which made somewhat uncertain the true origin of the detected X-ray emission. Finally, we found no radio counterparts corresponding to the five X-rays point sources detected using XMM-Newton observations at the location of the emission in $\gamma$ rays (Abramowski et al. 2014).

\section{Radio spectral analysis in SNR G15.4+0.1}

\subsection{The integrated spectrum}

To determine the global spectral index $\left(S \propto v^{\alpha}\right)$ of G15.4+0.1, we have used the new flux density estimate at $624 \mathrm{MHz}$ together with that obtained from GMRT data at $1420 \mathrm{MHz}(\mathrm{C} 13)$ and measurements at 330,1400 , and $2700 \mathrm{MHz}$ as determined from observations carried out with the VLA and Effelsberg telescopes (Brogan et al. 2006). In Table 1 we summarized the flux densities integrated over the whole extension of this remnant at the mentioned frequencies. The listed values constitute all the measurements published up to present for the source. A correction

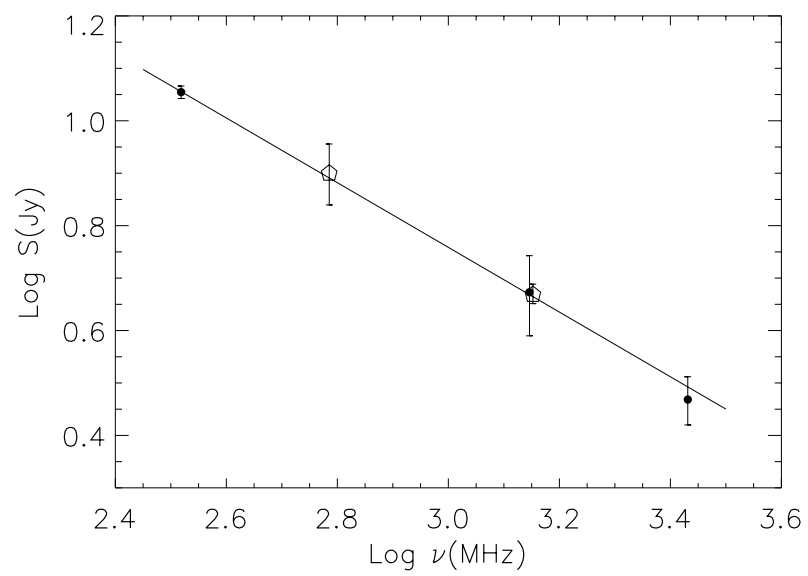

Fig. 5. Integrated radio continuum spectrum of SNR G15.4+0.1. The open symbols correspond to GMRT flux densities measurements at 624 and $1420 \mathrm{MHz}$. The rest of the data were taken from Brogan et al. (2006). All the data were tied to a common flux density scale (Perley \& Butler 2013). The solid line shows the best fit obtained with a single power law to all the values, which yields a global spectral index $\alpha=-0.62 \pm 0.03$.

factor was applied to set all the values to the Perley \& Butler (2013) absolute flux density scale. In particular, the correction to the flux density estimates at 330 and $624 \mathrm{MHz}$ was made using the low frequency extension proposed by those authors. Figure 5 shows the resulting radio continuum spectrum of the remnant; the determinations from GMRT observations are plotted as open pentagons symbols. A weighted fit to all the available flux densities yields a spectral index $\alpha=-0.62 \pm 0.03$, which is compatible with the value derived previously by Brogan et al. (2006). This spectral behaviour is consistent with that measured in other shell type SNRs (Kothes et al. 2006).

\subsection{Spatial variations in the radio spectral index}

We have investigated the spectral changes as a function of the position within the remnant by using the image at $624 \mathrm{MHz}$ and the one at $330 \mathrm{MHz}$ (see panel (f) in Fig. 6) kindly provided by Brogan (Brogan et al. 2006). The analysis was done by constructing tomographic maps between them (Katz-Stone \& Rudnick 1997). 

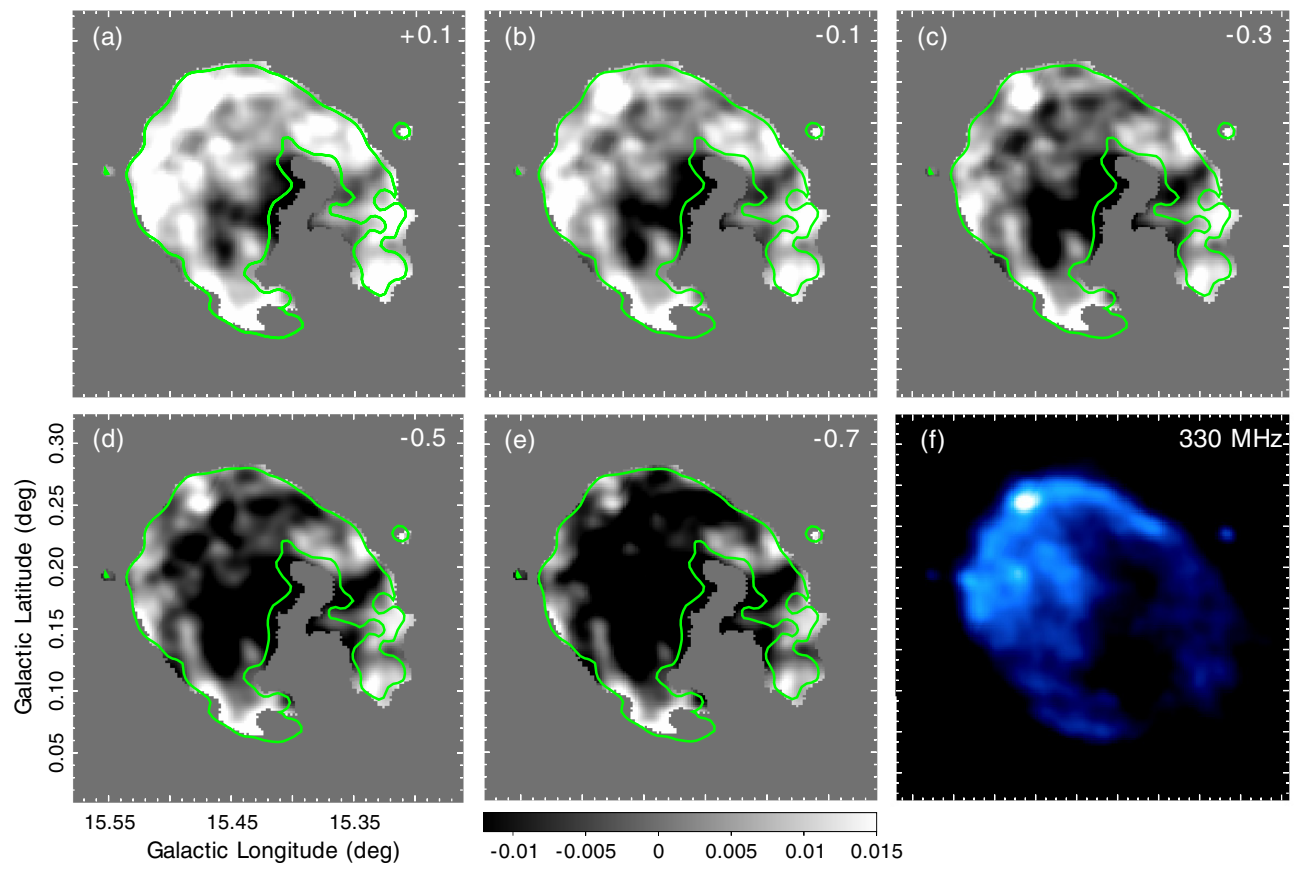

Fig. 6. Tomographic images for SNR G15.4+0.1 constructed from data at 330 and $624 \mathrm{MHz}$. The data at both frequencies were matched to the same $u v$-coverage. The radio spectral index $\alpha_{\mathrm{t}}$ is indicated at the top right corner of the panels. The grey colour bar in the middle column represents the scale of the residuals components, which is the same for all tomographies. In the greyscale representation, spectral components for which $\alpha$ is steeper than the assumed $\alpha_{\mathrm{t}}$ are shown as light regions (positive residuals), while flatter components are seen as dark features (negative residuals). A contour delineating the radio continuum emission at $330 \mathrm{MHz}$ is included for reference. The total intensity image of G15.4+0.1 at $330 \mathrm{MHz}$ is plotted in the bottom right frame (Brogan et al. 2006).

Basically, the procedure involves the generation of a series of residual images $S_{\mathrm{t}}$ according to the expression $S_{\mathrm{t}}=$ $S_{330}-\left(\frac{330}{624}\right)^{\alpha_{\mathrm{t}}} S_{624}$, where $S_{330}$ and $S_{624}$ are the maps at 330 and $624 \mathrm{MHz}$, respectively, and $\alpha_{\mathrm{t}}$ represents a trial spectral index value. If the assumed spectral index coincides with the "true" value, one should expect the $S_{\mathrm{t}}$ image to be equal to zero. On the contrary, features in the residual map that appear positive (negative) will be associated with local spectral indices that are steeper (flatter) than the assumed $\alpha_{\mathrm{t}}$ value. The technique has the advantage of delineating spectral features that can overlap along the line of sight while avoiding any zero level dependence.

In Fig. 6, we reproduce the gallery of tomographic maps between 330 and $624 \mathrm{MHz}$. To construct them, the radio images were clipped at the $3 \sigma$ level of their respective noise levels. In order to obtain a reliable determination of the spectral properties, the range of the spatial scales measured at $624 \mathrm{MHz}$ was matched in the $u v$-coverage to that of the $330 \mathrm{MHz}$ data. Additionally, the images were aligned and interpolated to identical projections to avoid positional offsets. The $\alpha_{\mathrm{t}}$ value shown in the maps varies from +0.1 to -0.7 . For the sake of illustration the $330 \mathrm{MHz}$ total intensity image of G15.4+0.1 is shown in the bottom right frame in Fig. 6.

Spatial variations in the spectral index are recognizable across the synchrotron emission from G15.4+0.1. The central part of the remnant shows components of spectral index flatter ( $\alpha$ varying between $\sim+0.1$ and -0.5 ) than the values in the ridge. We note that the very flat spectral component observed as a dark area in the southeastern border of the remnant $(l \sim 15: 40$, $b \sim 0$.15), within which the spectral index appears to have positive values (see panel corresponding to $\alpha_{\mathrm{t}}=+0.1$ in Fig. 6), corresponds to a bordering clipped area in the map and should not be considered a real spectral component owing to the decrease in the signal-to-noise ratio observed in this region in the VLA $330 \mathrm{MHz}$ image in comparison with the image at $624 \mathrm{MHz}$. In general, the central flattening traced in the $330 / 624 \mathrm{MHz}$ comparison matches the zone where the interaction with the discovered CO molecular cloud is taking place (see Fig. 4). Variations in the spectrum are generally expected in regions where the SN shock is impacting high density interstellar material as a consequence of the density increase and the deceleration of the shock in the interacting regions. For instance, spectral flattening were found between 74 and $330 \mathrm{MHz}$ for the two well-known SNRs/molecular clouds systems marking the ionized interface of molecular clouds interacting with the shock front in 3C 391 (Brogan et al. 2005b) and IC 443 (Castelletti et al. 2011).

The tomographies also show a conspicuous bullet-like feature standing out as a bright spectral component (i.e. positive in the $S_{\mathrm{t}}$ maps) located at the northeast part of the shell $\left(l \sim 15^{\circ} .48\right.$, $b \sim 0.25$ ). It is characterized by a spectral index even steeper than -0.7 because it appears as a positive residual in all tomographic images. We found no catalogued extragalactic objects at the position of this feature.

Along the westernmost edge (left side) of the SNR, there is an indication of a positive correlation between the local spectral index and the total radio intensity features in the sense that the bright ridge tends to be associated with steeper indices. A similar situation was found in young SNRs (see e.g. the analysis of SNRs G39.2-0.3 and G41.1-0.3 in Anderson \& Rudnick 1993). In those cases, it was suggested that the radio brightness and spectra are being regulated by different mechanisms. In addition to this behaviour, most of the structures in the tomographies traced at $\alpha_{\mathrm{t}}=-0.5$ and $\alpha_{\mathrm{t}}=-0.7$ disappear against the background indicating that the spectral behaviour can be characterized by a mean spectral index $\sim-0.6$, which is consistent with the integrated spectral index of this SNR derived 


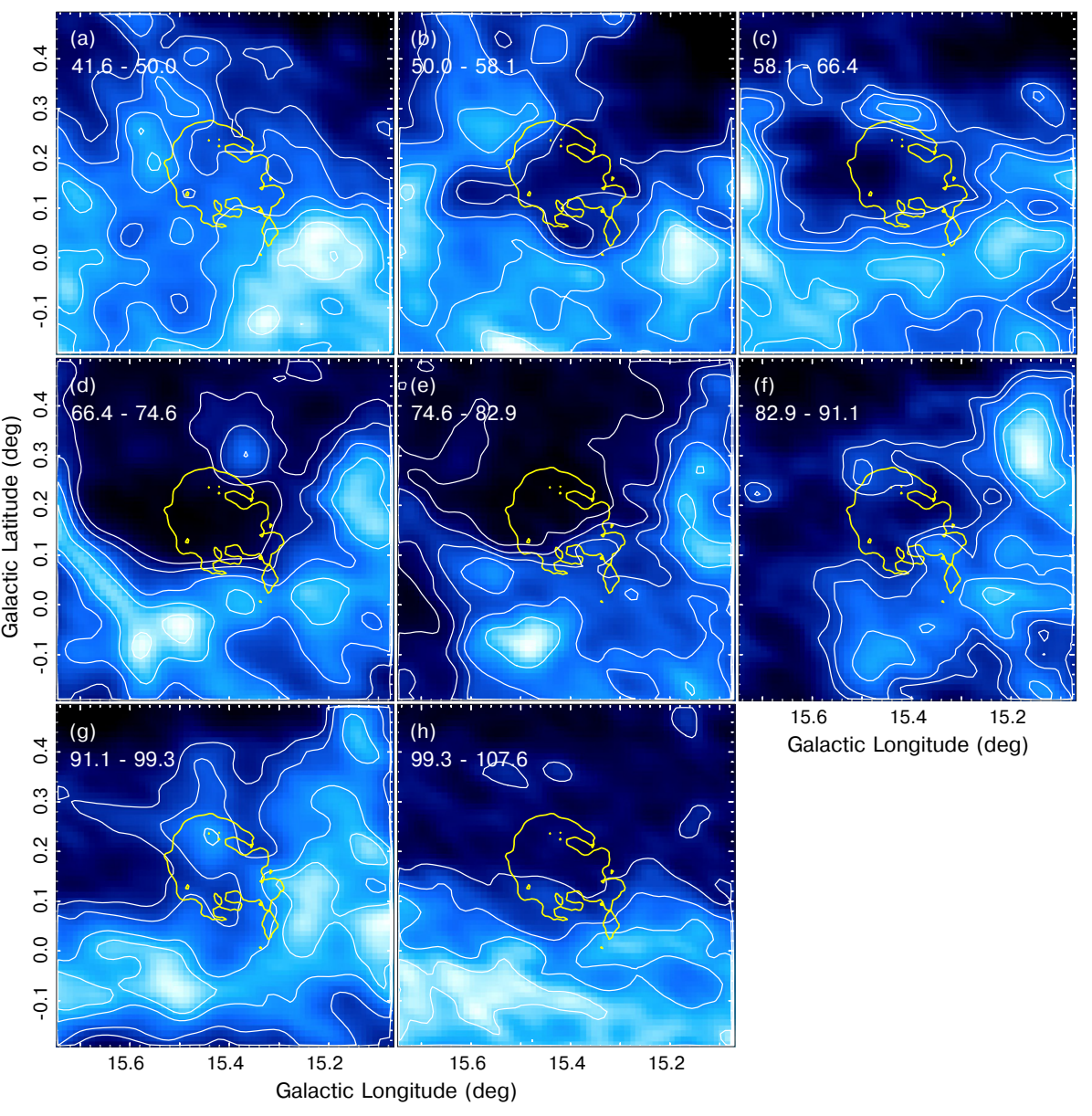

Fig. 7. Images of $21 \mathrm{~cm}$ line emission in the direction of SNR G15.4+0.1. Each map corresponds to the integration of the neutral gas every $8.2 \mathrm{~km} \mathrm{~s}^{-1}$. The range of velocities is indicated at the top right corner of the panels. The colour display was kept for all images between 633 and $1210 \mathrm{~K} \mathrm{~km} \mathrm{~s}^{-1}$. The $0.7 \mathrm{mJy}$ beam $^{-1}$ contour from the $35^{\prime \prime}$ resolution $624 \mathrm{MHz}$ image is included in each panel to facilitate the comparison between the radio continuum emission from the remnant and its surroundings. by fitting the total flux densities from 330 to $2700 \mathrm{MHz}$ (see Sect. 4.1). Overall, the observed radio continuum spectral distribution across G15.4+0.1 is compatible with what is expected in the framework of diffusive shock acceleration models.

\section{Neutral gas distribution around G15.4+0.1}

\subsection{HI brightness morphology and physical parameters}

We explored the ISM around G15.4+0.1 in the $21 \mathrm{~cm}$ line to distinguish some morphological correlations between the distribution of the neutral gas and the remnant that might account for the SNR shock effects on the surrounding medium.

Figure 7 includes velocity channels maps from the SGPS data cube covering an interval from 41.6 to $\sim 108 \mathrm{~km} \mathrm{~s}^{-1}$. All the velocities are measured with respect to the local standard of rest (LSR). Each frame was obtained by integrating the data cube every $8.2 \mathrm{~km} \mathrm{~s}^{-1}$. An appropriate background level equal to the mean value of each integrated image was subtracted from every map. A contour line delineating the outer boundary of the radio continuum emission at $624 \mathrm{MHz}$ from G15.4+0.1 was overlaid to the HI distribution for ease of comparison.

The distribution of the neutral gas in the velocity range from 41.6 to $50 \mathrm{~km} \mathrm{~s}^{-1}$ only shows bright clouds spread over the field without any correspondence with the radio continuum shell of G15.4+0.1. A similar behaviour is found between 0 and $41.6 \mathrm{~km} \mathrm{~s}^{-1}$ and therefore, the images are not shown. In the images corresponding to velocities between 50 and $91.1 \mathrm{~km} \mathrm{~s}^{-1}$, we observed the best morphological signature of neutral gas related to the SNR. Indeed, at $\sim 54 \mathrm{~km} \mathrm{~s}^{-1}$, the HI distribution appears to be distorted at the position of the synchrotron emission from G15.4+0.1. Moreover, it is in the $58.1-91.1 \mathrm{~km} \mathrm{~s}^{-1}$ range where we see a cavity in the $\mathrm{HI}$ emission that closely matches G15.4+0.1. In particular, at velocities from 58.1 to $74.6 \mathrm{~km} \mathrm{~s}^{-1}$, the HI distribution has an almost complete shelllike morphology along the eastern and western peripheries (left and right sides) of the remnant, which looks broken toward the northeastern border. The presence of this shell is compatible with the central systemic velocity $v_{\text {sys }}=60 \mathrm{~km} \mathrm{~s}^{-1}$ derived in $\mathrm{C} 13$ for the SNR. The expanding shock wave of the SNR on the HI surroundings might be responsible for the creation of the observed HI structure. An alternative origin for the HI shell may be a preexisting stellar wind bubble formed for instance by a group of OB stars, one of which could have been the SN precursor of G15.4+0.1. In this particular scenario, the brightest synchrotron emission observed towards the northwest face of the remnant could be where the shock is interacting with the inner wall of the bubble. Furthermore, the fact that ${ }^{13} \mathrm{CO}$ emission was detected on the shell's central face of G15.4+0.1 at approximately $50 \mathrm{~km} \mathrm{~s}^{-1}$ (C13) may indicate that the molecular cloud is outside the front wall of atomic hydrogen; perhaps the inner wall is also the place where the synchrotron emission originates. Examples of bubbles created by the action of the precursor's stellar winds, inside of which evolves a SNR shock wave, are those discovered surrounding the remnants Kes 79 (Giacani et al. 2009), 3C 434.1 (Foster et al. 2004), and G106.3+2.7 (Kothes et al. 2001). In any case, whether the HI shell is formed by neutral gas accelerated by the SNR shock or by stellar winds from the SN progenitor, the X-ray emission detected inside the radio shell of the SNR reinforces the idea that the center has been evacuated. It is 
also worth noting that at higher velocities there is no evidence of the naturally expected cap-like features associated with an HI shell. The non-detection of possible caps does not imply that these structures do not exist. They could be undetected because of confusion caused by unrelated foreground and background inhomogeneities in the ISM. The caps, produced by accelerated HI gas, have been observed either in emission as central concentrations projected within the remnant (Velázquez et al. 2002) or in HI self-absorption in an expanding stellar wind bubble wall (Foster et al. 2004). At velocities higher than $\sim 91.1 \mathrm{~km} \mathrm{~s}^{-1}$, the $21 \mathrm{~cm}$ line emission does not correlate with the SNR, and neutral gas is preferentially oriented parallel to the Galactic plane.

In what follows, we derive a number of parameters characterizing the HI shell. For this structure, centered at $l \sim 15.42$, $b \sim 0$.16, we assigned a radius of $\sim 9.8$ or $\sim 13.7$ pc at a distance of $4.8 \mathrm{kpc}$. If we assume that the HI emission is optically thin, the integration for velocities between 50 and $91 \mathrm{~km} \mathrm{~s}^{-1}$, for which we observed the most evident signatures of association between the HI gas and the SNR, yields an average column density $N_{\mathrm{H}} \simeq 4 \times 10^{20} \mathrm{~cm}^{-2}$. The total mass of atomic gas that forms the shell is about $1.9 \times 10^{3} M_{\odot}$. This value is in fact an upper limit due to the impossibility of disentangling related and unrelated HI gas. If the HI shell was formed by ISM swept up by the SNR shock and assuming that before the SN event the total neutral mass was uniformly distributed inside the volume of the HI shell, the derived mean density of hydrogen nuclei in the ambient environment is $\eta_{0} \sim 7 \mathrm{~cm}^{-3}$, which is larger than the typical interstellar hydrogen densities of $\sim 1 \mathrm{~cm}^{-3}$ (averaged over the cold, warm, and hot gas-phase constituents of the ISM, McKee $\&$ Ostriker 1977). Our result corresponds to a reasonable kinetic energy supplied by the SN explosion into the the surrounding medium of about $7.6 \times 10^{48} \mathrm{erg}$. This value was estimated by adopting an expansion velocity for the HI shell of $\sim 20 \mathrm{~km} \mathrm{~s}^{-1}$, based on the systemic velocity of the neutral gas derived in $\mathrm{C} 13$ $\left(v_{\text {sys }} \sim 60 \mathrm{~km} \mathrm{~s}^{-1}\right)$ and the HI-continuum correspondences seen between $\sim 50$ and $91 \mathrm{~km} \mathrm{~s}^{-1}$. Taking into account errors in the selection of the boundaries for integration, confusion from background or foreground unrelated HI gas, as well as uncertainties in the determination of the distance, we derived the parameters for the HI shell with a mean relative error of $45 \%$.

As a by-product, on the basis of the new radio continuum image at $624 \mathrm{MHz}$ and the results derived from the $\mathrm{HI}$ data, we calculated physical parameters of the remnant G15.4+0.1. By combining the ambient density $\eta_{0} \sim 7 \mathrm{~cm}^{-3}$, the radius of G15.4+0.1 $R_{\mathrm{SNR}} \sim 7.2(\sim 10.1 \mathrm{pc}$ at the distance of $4.8 \mathrm{kpc})$ with the velocity of the shock, $v_{\text {sh }}$, we can roughly calculate the energy released by the SN explosion to the ISM from the expression given in the model discussed by Chevalier (1974),

$E_{\mathrm{SN}}=5.3 \times 10^{43} \eta_{0}^{1.12} v_{\mathrm{sh}}^{1.40} R_{\mathrm{SNR}}^{3.12} \mathrm{erg}$.

To estimate the shock velocity, we used $v_{\mathrm{sh}}=$ $66.5\left(\frac{R_{\mathrm{SNR}}}{21.9}\right)^{-2.23} \mathrm{~km} \mathrm{~s}^{-1}$ and obtained $v_{\mathrm{sh}}=370 \mathrm{~km} \mathrm{~s}^{-1}$ for a shock radius set to $R_{\mathrm{SNR}} \sim 10.1 \mathrm{pc}$. We understand that this value represents a reliable bound for the velocity of the SN shock before impacting the dense molecular cloud located in front of G15.4+0.1; the velocity range of $46-50.3 \mathrm{~km} \mathrm{~s}^{-1}$ for the cloud corresponds to a distance of $\sim 4.2 \mathrm{kpc}(\mathrm{C} 13)$. To test the robustness of our interpretation, we also estimated the shock velocity using the physical parameters derived for the ${ }^{13} \mathrm{CO}$ cloud interacting with SNR G15.4+0.1, as calculated with the expression $v_{\mathrm{sh}}=v_{\mathrm{cl}}\left(\eta_{\mathrm{cl}} / \eta_{\text {int }}\right)^{1 / 2}$, where $\eta_{\mathrm{cl}}$ and $v_{\mathrm{cl}}$ are the density and the expansion velocity of the cloud, respectively, and $\eta_{\text {int }}$ is the intercloud density assumed to be
$1 \mathrm{~cm}^{-3}$ (Dubner et al. 1999). In C13, it was demonstrated that the $\mathrm{CO}$ cloud interacting with $\mathrm{G} 15.4+0.1$ has a density of $\sim 1.5 \times 10^{3} \mathrm{~cm}^{-3}$ and it is expanding at $\sim 10 \mathrm{~km} \mathrm{~s}^{-1}$. In these circumstances, we derived a shock velocity of $v_{\mathrm{sh}} \simeq 380 \mathrm{~km} \mathrm{~s}^{-1}$, which agrees within the uncertainties of the method with that obtained using HI data. Finally, for an average shock velocity of $375 \mathrm{~km} \mathrm{~s}^{-1}$ and using the ambient density along with the radius of the shell derived from our study, we obtain an initial energy of $\sim 2.6 \times 10^{51} \mathrm{erg}$, which agrees with the standard value.

To account for the age of G15.4+0.1, we employed the dynamical evolution model of Chevalier (1974) and obtained $t \simeq\left(\frac{R_{\mathrm{SNR}}}{21.9}\right)^{3.23} 10^{5} \simeq 8.2 \times 10^{3} \mathrm{yr}$, where we used a radius for the SNR of $R_{\text {SNR }} \sim 10.1 \mathrm{pc}$ at a distance of about $4.8 \mathrm{kpc}$. This result differs from that calculated by Abramowski et al. (2014) $\left(2.5 \times 10^{3} \mathrm{yr}\right)$ based on a Sedov-Taylor and the assumption of an ambient density around G15.4+0.1 of $1 \mathrm{~cm}^{-3}$.

\subsection{Modelling the broadband spectrum of G15.4+0.1}

The analysis in Abramowski et al. (2014) to determine the origin of the $\gamma$-ray emission in the region of G15.4+0.1 excludes a hadronic contribution. In modelling the spectral energy distribution (SED), these authors used radio data at 330 and $1400 \mathrm{MHz}$ from the GPS (Brogan et al. 2005a) and the Multi-Array Galactic Plane Imaging Survey (MAGPIS, Helfand et al. 2006), respectively, whose fluxes were treated as upper limits, X-ray data from XMM-Newton, and $\gamma$-ray observations obtained with H.E.S.S. In this section, we first demonstrate that, with the available data, a hadronic model in which the $\mathrm{TeV} \gamma$-ray flux is produced through the interaction of accelerated protons with ambient protons, mainly from the molecular cloud located foreground $\mathrm{G} 15.4+0.1$, is still compatible with the broadband spectrum of the G15.4+0.1/HESS J1818-154 system. In addition, in the light of the new GMRT data, we revisited the leptonic model presented in Abramowski et al. (2014) to fit the multiwavelength spectral data.

The energy spectrum of the accelerated particles (electrons and protons) in the emission region is described by a power law with an exponential cut-off,

$\frac{\mathrm{d} n_{\mathrm{e}, \mathrm{p}}}{\mathrm{d} E}=K_{\mathrm{e}, \mathrm{p}} E^{-\Gamma_{\mathrm{e}, \mathrm{p}}} \exp \left(-E / E_{\mathrm{cut}_{\mathrm{e}, \mathrm{p}}}\right)$,

where the subscripts e and p refer to the particle species (electrons or protons), and $E, K, \Gamma$, and $E_{\text {cut }}$ are the particle energy, a normalization constant, the spectral index, and the cut-off energy, respectively. We refer to Aharonian et al. (2010) and references therein for calculation details of the synchrotron emissivity produced by electrons. Regarding the inverse Compton (IC) and the non-thermal Bremsstrahlung emission, we followed the method given in Jones (1968) for the former, while the method presented in Baring et al. (1999) was used to model the electronelectron Bremsstrahlung interaction and in Koch \& Motz (1959) and Sturner et al. (1997) for the electron-ion Bremsstrahlung process. On the other hand, we determine the $\gamma$-ray spectrum due to the neutral pion decays by using the ppfrag program (Kachelrieß \& Ostapchenko 2012). In the calculations, the low and high energy parts of the spectrum were separately considered. For energies of the $\gamma$ rays smaller than $50 \mathrm{GeV}$, we used the parametrization presented in Kamae et al. (2007), while for larger values of energy we based our calculations in the interaction model QGSJET-II-049 (Ostapchenko 2011).

9 The last version of the QGSJET model was updated with recent Large Hadron Collider data. 


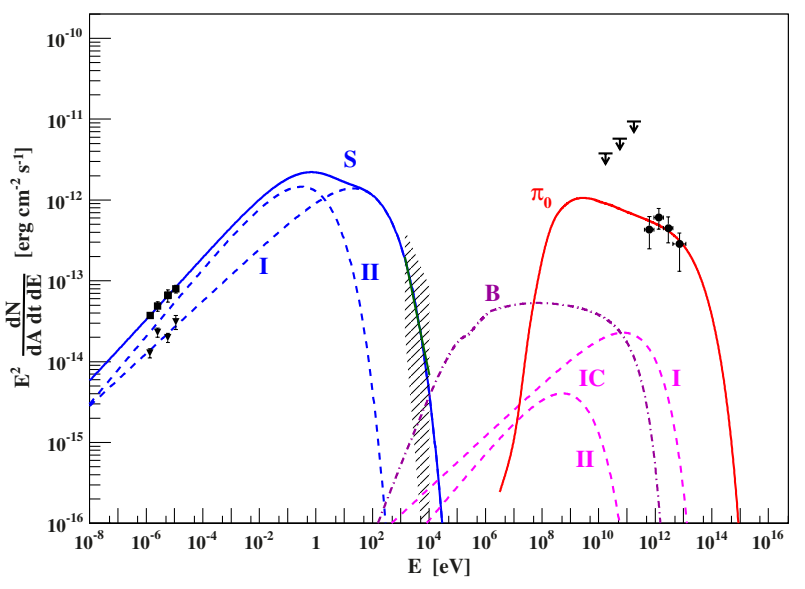

Fig. 8. Spectral energy distribution of the SNR G15.4+0.1. The filled squares and triangles with error bars correspond to the emission from the whole remnant and from the interior of the shell (Region I), respectively (data from Brogan et al. 2006, and this work). The shadowed region encompassing a solid line corresponds to the fitted power law and the one sigma region of the X-ray data. The HESS TeV data points are indicated by filled circles (Abramowski et al. 2014), while the arrows pointing downwards show the upper limits in the Fermi-LAT spectrum (Acero et al. 2013). The dashed lines labelled I and II mark the spectra from Region I, assuming non-thermal X-ray and radio emission originating in the interior of G15.4+0.1, and from Region II in the shell of the remnant, respectively. The modelled spectra from the synchrotron radiation by electrons, inverse Compton on the CMB, Bremsstrahlung, and $\gamma$ rays produced by neutral pions generated in proton-proton interactions are indicated by the curves labelled $\mathrm{S}, \mathrm{IC}, \mathrm{B}$, and $\pi_{0}$, respectively.

The XMM-Newton observations show faint X-ray emission, about 9 arcmin in size, which partially coincides with the size of the HESS J1818-154 source (see Fig. 4). The origin of the $\mathrm{X}$-rays was considered non-thermal and based on their spatial correspondence with the $\gamma$ rays, an association between them was suggested (Abramowski et al. 2014). We model the spectrum of $\mathrm{G} 15.4+0.1$ from radio to the $\mathrm{TeV} \gamma$ rays by considering a non-thermal nature of the X-ray emission. This implies that the whole X-ray flux and part of the radio flux is produced by synchrotron radiation of electrons in the turbulent magnetic field in the region interior to the SNR shell, the PWN proposed in Abramowski et al. (2014). We refer to this zone as Region I. The remaining part of the radio flux is also generated by synchrotron radiation, but corresponding to a different population of electrons that are placed in the shell and referred to hereafter as Region II. Figure 8 shows the broadband spectrum for the SNR G15.4+0.1/HESS J1818-154 system. The radio data for Regions I and II include the observations presented in Brogan et al. (2006) and the new GMRT data at 624 and $1420 \mathrm{MHz}$. The spectrum also includes the best fit to the X-ray data using a power law taken from Abramowski et al. (2014), and upper limit $\gamma$-ray fluxes obtained by Fermi-LAT (Acero et al. 2013) and HESS (Abramowski et al. 2014). Although upper limits on the fluxes at $\mathrm{GeV}$ energies are represented, they were not used in fitting the spectrum as they are too far from HESS data. The best fit to the radio data corresponding to the interior SNR region is shown by a dashed curve labelled I. The intensity of the magnetic field and the cut-off energy are fixed during the fitting procedure in order to be consistent with the power-law fit to the X-ray data. The normalization constant $K$ and the spectral index $\Gamma$ in Eq. (2) are taken as free fit parameters. The intensity of the magnetic field used is $B^{\mathrm{I}}=25 \mu \mathrm{G}$ and the cutoff energy is $\log \left(E_{\text {cut,e }}^{\mathrm{I}} / \mathrm{eV}\right)=12.83$. These values are chosen in such a way that the inverse Compton component has a subdominant role at very high energies. It is worth mentioning that scenarios with larger values of the magnetic field intensity and smaller cut-off energies of the electron component, such that $\left(E_{\text {cut }, \mathrm{e}}^{\mathrm{I}}\right)^{2} \times B^{\mathrm{I}}=1.1536 \times 10^{21} \mathrm{eV}^{2} \mathrm{G}$, also describe properly the X-ray data, making the inverse Compton component even smaller. The best-fit model for Region I yields a spectral index $\Gamma_{\mathrm{e}}^{\mathrm{I}}=2.35 \pm 0.14$.

We also considered a contribution to the leptonic mechanism from accelerated electrons in the SNR shell. The radio flux, corresponding to the shell region, is obtained by fitting the total radio flux, by using the best fit to the radio data corresponding to the internal region. In this case, we fixed the cut-off energy and the intensity of the magnetic field to $\log \left(E_{\text {cut,e }}^{\mathrm{II}} / \mathrm{eV}\right)=11.6$ and $B^{\mathrm{II}}=62.5 \mu \mathrm{G}$, respectively. This choice of the parameters prevents the synchrotron flux that originated in the shell region from contributing to the X-ray part of the SED. We note that this combination of the two parameters is not unique. Smaller values of the cut-off energy, which are consistent with the data, produce smaller values of the high energy flux originated by the inverse Compton process. The spectral index obtained from the fit is $\Gamma_{\mathrm{e}}^{\mathrm{II}}=2.16 \pm 0.08$. It is evident that in our model the inverse Compton process alone cannot explain the emission at $\mathrm{TeV}$ energies from Region I or II. Figure 8 also includes the spectrum corresponding to the Bremsstrahlung radiation. In this case, we assumed that only Region II has enough density to produce a non-negligible flux. The calculation was done by fixing the proton density, both molecular and atomic, to the value $n=6.4 \mathrm{~cm}^{-3}$ averaged over Region II. We note that, as in the IC model, it is possible to reduce even more the Bremsstrahlung effects diminishing the value of the cut-off energy of the accelerated electrons of the shell region.

It is worth mentioning that the magnetic field intensity of the two regions required to describe the data is much larger than the field corresponding to the ISM, for which its typical strength is 1-2 $\mu \mathrm{G}$ (Foster et al. 2013, and references therein). This is typical in hadronic models of the $\gamma$-ray emission (see e.g. Cardillo et al. 2014).

We determined the hadronic contribution of the $\gamma$-ray flux resulting from the neutral pion decay of accelerated protons (illustrated by a solid line labelled as $\pi^{0}$ in Fig. 8). Since neither the HESS data nor the Fermi-LAT upper limits are enough to constrain the spectral index of the proton component, we used the spectral index of the electron component fitted for the synchrotron emission in Region II, that is $\Gamma_{\mathrm{p}}^{\mathrm{II}}=2.16$. Taking into account the normalization constant and the cut-off energy as free fit parameters, we obtained for the proton component a cut-off energy $\log \left(E_{\text {cut }, \mathrm{p}} / \mathrm{eV}\right)=14.6 \pm 0.5$.

On the other hand, we revisited the one-zone model presented in Abramowski et al. (2014) replacing the upper limits on the radio fluxes considered in that work by reliable flux density measurements of the radio synchrotron emission in the interior of G15.4+0.1 (called Region I in the current work). This region is spatially coincident with the X-ray emission attributed by those authors to the same electron population responsible for the $\gamma$-ray emission through the IC process. In this case as well, a power law with an exponential cut-off was assumed to model the spectral energy distribution of electrons. As was done for the hadronic scenario, we imposed the condition $\left(E_{\text {cut }, \mathrm{e}}\right)^{2} \times B=1.1536 \times 10^{21} \mathrm{eV}^{2} \mathrm{G}$, which ensures an adequate description of the X-ray data. We would like to note that also in this case the Fermi-LAT upper limits do not constrain the model. The corresponding spectrum is shown in Fig. 9. For this 


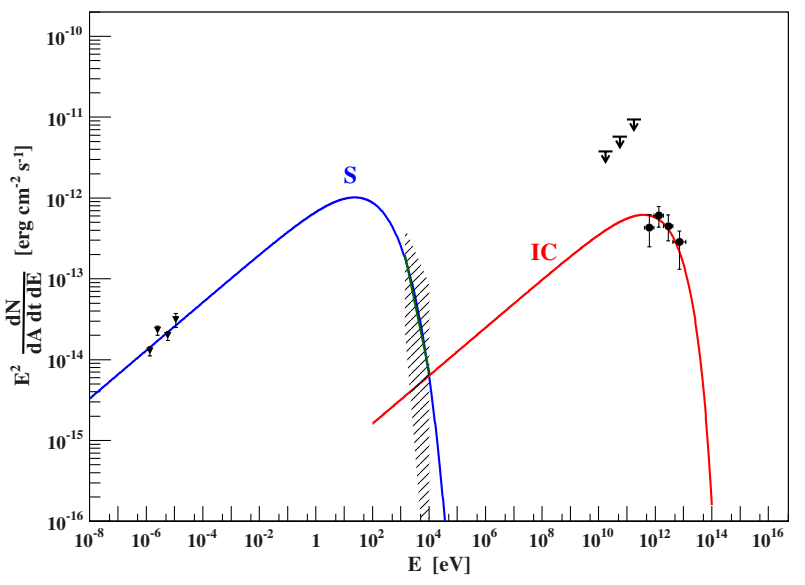

Fig. 9. Broadband fit to radio (filled triangles) and $\gamma$-ray observations obtained with HESS (filled circles). Upper limits to the flux densities from Fermi-LAT (arrows pointing downwards) and the best fit to the X-ray data (green hatching) are also included. The radio data correspond to the internal region of SNR G15.4+0.1. The curves labelled $\mathrm{S}$ and IC show the model of the synchrotron radiation and inverse Compton mechanism on the CMB by accelerated electron, respectively.

simple model, we obtain a spectral index $\Gamma_{\mathrm{e}}=2.41 \pm 0.09$, a magnetic field $B=3.9 \pm 1.4 \mu \mathrm{G}$, and a cut-off energy of electrons $\log \left(E_{\text {cut,e }} / \mathrm{eV}\right)=13.303 \pm 0.071$. The fit corresponding to this scenario is as good as the one obtained in Abramowski et al. (2014). However, the fitted parameters derived in this work are restricted to smaller intervals. This is due to the radio data points of the internal region used to fit the SED.

The observational data is compatible with both scenarios analyzed here; more data is needed, especially in the energy region relevant to the Fermi-LAT observatory, in order to make progress in the understanding of the most important physical processes taking place in this source. In any case, it is important to emphasize that a hadronic scenario is compatible with the observational data.

\section{Conclusions}

We presented new full-synthesis imaging of the SNR G15.4+0.1 obtained from observations at $624 \mathrm{MHz}$ carried out with the GMRT. We measured an integrated flux density $S=8.0 \pm 1.1 \mathrm{Jy}$ at $624 \mathrm{MHz}$. Based on the combination of our estimate with those previously published, after bringing all values to the same absolute flux density scale, we derive a spectral index $\alpha=$ $-0.62 \pm 0.03$ for the whole remnant. The estimated global spectral index is consistent with measurements for other typical SNR shells (Kothes et al. 2006).

The new GMRT data were used to analyze the correspondences with FIR and X-ray observations towards the remnant. We found an impressive correlation between the infrared emission detected by Herschel at 70 and $250 \mu \mathrm{m}$ and the cloud of ${ }^{13} \mathrm{CO}$ colliding with the SNR shock reported in C13. In other regions of G15.4+0.1, however, no IR counterpart is observed to the radio emission. On the other hand, from the comparison of the new $624 \mathrm{MHz}$ image with re-processed XMM-Newton observations between 1 and $8 \mathrm{keV}$, we can confirm that the synchrotron radio emission surrounds the low surface brightness X-ray radiation. No counterparts were observed in radio to the five point-like sources detected in the XMM-Newton field. We also conducted a search for a neutron star associated with
G15.4+0.1 through time-series observations performed with the GMRT, which provided negative results.

The analysis of the spatial spectral index variations made between existing observations of G15.4+0.1 at 330 and the new GMRT data at $624 \mathrm{MHz}$ revealed a radio spectrum steepening from the weak interior to the brighter periphery of G15.4+0.1. A similar spectral behaviour is also found in other young SNRs. The overall distribution of the local spectral radio index could be described by diffusive shock acceleration by considering the effect of the ISM gas. We did not recognize any distinct feature in the radio brightness at $624 \mathrm{MHz}$ or in the 330/624 spectral distribution that could be considered a synchrotron nebula, powered by the wind of a yet undetected pulsar. Furthermore, our search for radio pulsations inside the synchrotron shell did not reveal a pulsar, with the upper limits on the mean flux density of any associated putative pulsar of 250 and $300 \mu \mathrm{Jy}$ at 624 and $1404 \mathrm{MHz}$, respectively, towards the centroid of the TeV source. In addition, the flux density upper limits that we estimated for any putative pulsar morphologically coincident with SNR G15.4+0.1, including the five point-like X-ray sources reported by Abramowski et al. (2014), were of about 700 and $500 \mu \mathrm{Jy}$ at 624 and $1404 \mathrm{MHz}$, respectively .

Our study of the neutral ambient gas in the direction of $\mathrm{G} 15.4+0.1$ unveiled a large (about $13.7 \mathrm{pc}$ in radius at a distance of $4.8 \mathrm{kpc}$ ) and incomplete HI shell-type structure. We determined a mass for this shell of $1.9 \times 10^{3} M_{\odot}$ and a kinetic energy of $7.6 \times 10^{48} \mathrm{erg}$. On the basis of this study and the new SNR image at $624 \mathrm{MHz}$, we estimated that G15.4+0.1 was created as the result of $2.6 \times 10^{51} \mathrm{erg}$ released in a $\mathrm{SN}$ explosion that occurred about $8.2 \times 10^{3}$ yr ago.

Finally, we discussed different models to fit the broadband emission from the SNR G15.4+0.1/HESS J1818-154 system. In particular, we demonstrated that taking into consideration the multiwavelength data known to date, a purely hadronic picture is sufficient to account for the $\gamma$-ray data. This is in contrast to the explanation given in Abramowski et al. (2014) for which the $\mathrm{X}$-ray and $\mathrm{TeV}$ emission have the same origin. We recognize that more comprehensive X-ray and $\gamma$-ray data (especially in the $\mathrm{MeV}-\mathrm{GeV}$ band) are required to improve the constraints on the observed SED of the system and distinguish between leptonic and hadronic explanations for the origin of HESS J1818-154.

Acknowledgements. We wish to acknowledge the referee for his very fruitful comments that improved our work. We thank C. Brogan for supplying us with the VLA $330 \mathrm{MHz}$ image. This research has been funded by Argentina grants ANPCYT-PICT 0795/08 and 0571/11. G.C. is Member of the Carrera of Investigador Científico of CONICET, Argentina. L.S. is a Ph.D. Fellow of CONICET, Argentina. The GOODS-Herschel data was accessed through the Herschel Database in Marseille (HeDaM - http://hedam. lam.fr) operated by CeSAM and hosted by the Laboratoire d'Astrophysique de Marseille. We thank the staff of the GMRT, who have made these observations possible. GMRT is operated by the National Centre for Radio Astrophysics of the Tata Institute of Fundamental Research. This work made use of the High Performance Computing facility, funded by grant 12-R\&D-TFR-5.02-0711, at National Centre for Radio Astrophysics of the Tata Institute of Fundamental Research.

\section{References}

Abramowski, A., Aharonian, F., Ait Benkhali, F., et al. 2014, A\&A, 562, A40 Acero, F., Ackermann, M., Ajello, M., et al. 2013, ApJ, 773, 77

Aharonian, F. A., Kelner, S. R., \& Prosekin, A. Y. 2010, Phys. Rev. D, 82, 043002 Anderson, M. C., \& Rudnick, L. 1993, ApJ, 408, 514

Anderson, L. D., Bania, T. M., Balser, D. S., \& Rood, R. T. 2011, VizieR Online Data Catalog: J/ApJS/194/32

Baring, M. G., Ellison, D. C., Reynolds, S. P., Grenier, I. A., \& Goret, P. 1999, ApJ, 513, 311 
Brogan, C. L., Gaensler, B. M., Gelfand, Y., et al. 2005a, in X-Ray and Radio Connections, eds. L. O. Sjouwerman, \& K. K. Dyer, 4

Brogan, C. L., Lazio, T. J., Kassim, N. E., \& Dyer, K. K. 2005b, AJ, 130, 148

Brogan, C. L., Gelfand, J. D., Gaensler, B. M., Kassim, N. E., \& Lazio, T. J. W. 2006, ApJ, 639, L25

Cañellas, A., Joshi, B. C., Paredes, J. M., et al. 2012, A\&A, 543, A122

Cardillo, M., Tavani, M., Giuliani, A., et al. 2014, A\&A, 565, A74

Castelletti, G., Dubner, G., Clarke, T., \& Kassim, N. E. 2011, A\&A, 534, A21

Castelletti, G., Supan, L., Dubner, G., Joshi, B. C., \& Surnis, M. P. 2013, A\&A, 557, L15 (C13)

Chevalier, R. A. 1974, ApJ, 188, 501

Cordes, J. M., \& Lazio, T. J. W. 2002 [arXiv:astro-ph/0207156]

Dubner, G., Giacani, E., Reynoso, E., et al. 1999, AJ, 118, 930

Foster, T., Routledge, D., \& Kothes, R. 2004, A\&A, 417, 79

Foster, T., Kothes, R., \& Brown, J. C. 2013, ApJ, 773, L11

Giacani, E., Smith, M. J. S., Dubner, G., et al. 2009, A\&A, 507, 841

Griffin, M. J., Abergel, A., Abreu, A., et al. 2010, A\&A, 518, L3

Helfand, D. J., Becker, R. H., White, R. L., Fallon, A., \& Tuttle, S. 2006, AJ, 131,2525

Hines, D. C., Rieke, G. H., Gordon, K. D., et al. 2004, ApJS, 154, 290

Hofverberg, P., Chaves, R. C. G., Méhault, J., \& de Naurois, M. 2011, in International Cosmic Ray Conference, 7, 247
Jones, F. C. 1968, Phys. Rev., 167, 1159

Kachelrieß, M., \& Ostapchenko, S. 2012, Phys. Rev. D, 86, 043004

Kamae, T., Karlsson, N., Mizuno, T., Abe, T., \& Koi, T. 2007, ApJ, 662, 779

Katz-Stone, D. M., \& Rudnick, L. 1997, ApJ, 479, 258

Koch, H. W., \& Motz, J. W. 1959, Rev. Mod. Phys., 31, 920

Kothes, R., Uyaniker, B., \& Pineault, S. 2001, ApJ, 560, 236

Kothes, R., Fedotov, K., Foster, T. J., \& Uyanıker, B. 2006, A\&A, 457, A1081

McClure-Griffiths, N. M., Dickey, J. M., Gaensler, B. M., et al. 2005, ApJS, 158, 178

McKee, C. F., \& Ostriker, J. P. 1977, ApJ, 218, 148

Ostapchenko, S. 2011, Phys. Rev. D, 83, 014018

Perley, R. A., \& Butler, B. J. 2013, ApJS, 204, 19

Sandberg, A., \& Sollerman, J. 2009, A\&A, 504, 525

Slane, P., Helfand, D. J., Reynolds, S. P., et al. 2008, ApJ, 676, L33

Sturner, S. J., Skibo, J. G., Dermer, C. D., \& Mattox, J. R. 1997, ApJ, 490, 619

Supan, L., Castelletti, G., Dubner, G., Surnis, M. P., \& Joshi, B. C. 2014, Boletin de la Asociacion Argentina de Astronomia (La Plata, Argentina), 56, 315

Turner, M. J. L., Abbey, A., Arnaud, M., et al. 2001, A\&A, 365, L27

Velázquez, P. F., Dubner, G. M., Goss, W. M., \& Green, A. J. 2002, AJ, 124, 2145

Zajczyk, A., Gallant, Y. A., Slane, P., et al. 2012, A\&A, 542, A12

Zharikov, S. V., Zyuzin, D. A., Shibanov, Y. A., \& Mennickent, R. E. 2013, A\&A, 554, A120 